\title{
Téoros
}

Revue de recherche en tourisme

\section{Du tourisme écoculturel en Belgique ?}

\section{André Hut}

Volume 18, numéro 1, printemps 1999

Les jardins du tourisme

URI : https://id.erudit.org/iderudit/1072301ar

DOI : https://doi.org/10.7202/1072301ar

Aller au sommaire du numéro

Éditeur(s)

Université du Québec à Montréal

ISSN

0712-8657 (imprimé)

1923-2705 (numérique)

Découvrir la revue

Citer cet article

Hut, A. (1999). Du tourisme écoculturel en Belgique ? Téoros, 18(1), 52-56.

https://doi.org/10.7202/1072301ar d'utilisation que vous pouvez consulter en ligne.

https://apropos.erudit.org/fr/usagers/politique-dutilisation/ 


\section{DU TOURISME ÉCOCULTUREL EN BelgiQue?}

\section{André Hut}

Compte tenu d'un terreau favorable qui explique à la fois l'abondance et la fragilité des parcs et des jardins en Belgique, le rappel de quelques événements jalonneront la lente évolution, durant un quart de siècle, en faveur de leur integration dans l'offre touristique. Un florilège d'échantillons en illustrera la variété et la qualité indéniables, susceptibles de répondre aux souhaits des visiteurs en quête du "paradis perdu $\%$

\section{UN TERREAU FAVORABLE}

En effet, ce petit pays de $30000 \mathrm{~km}^{2}$, jouit d'une terre fertile, bien irriguée par de nombreuses rivières et traversée par des fleuves importants, et d'un climat tempéré propice à la culture de légumes, de fleurs, de fruits et d'arbres de toute sorte. La population s'est développée, au cours du bimillénaire bientôt fêté, d'une manière de plus en plus dense. Sa situation géographique, coincée aux marches de plusieurs empires politiquement forts et successivement envahissants, a provoqué la multiplication de châteaux et d'enceintes pour abriter les petites et les grosses autorités politiques. Ces constructions coexistent à quelques kilomètres les unes des autres dans une juxtaposition et un enchevêtrement inextricables, morcellement du territoire consécutif à la féodalisation. La conversion à la foi chrétienne a multiplié Evêtchés et paroisses, abbayes, couvents et béguinages. Ces derniers ont bénéficié des dons et du mécénat des empereurs, des princesses et des régentes, expliquant la prolifération de parcs et jardins agrémentés et richement décorés d' cuvres-d'art des plus raffinées.

Il ne faudrait pas méconnaître un savoirfaire, véhiculé par les familles, pour faire fructifier les petits jardins domestiques, ni les compétences professionnelles des ingénieurs, des architectes et des jardiniers pour dessiner, aménager et entretenir les pares d'agrément situés aux pieds des résidences aristocratiques.

Enfin, au cours des deux derniers siècles, les entreprises d'horticulteurs et de pépiniéristes ont acquis une renommée bien audelà du territoire belge et ont même émigné au Québec et en Ontario, la formation à ces métiers ayant été assurée par des écoles professionnelles de qualité, notamment en architecture paysagère.

\section{PATRIMOINE FRAGILE ET VULNÉRABLE}

En contrepartie, les envahisseurs du premier millénaire, les combats incessants entre les puissants empereurs voisins dont la Belgique constituait le champ de bataille, les guerres de religion animees de rages iconoclastes et, enfin, la Révolution française ont endommagé, incendié, détruit châteaux, abbayes et évêchés symbolisant l'Ancien Régime, ou affecté ceux-ci à $\mathrm{d}^{\prime}$ autres fonctions commerciales, industrielles, caritatives ou éducatives. Quant aux pares et aux jardins, ils ont été majoritairement laissés à l'abandon, saccagés, empiétés par des routes ou des chemins de fer, lotis pour la construction de résidences ou d'entreprises.

Dépourvus d'un entretien que les rythmes saisonniers contraignent à des soins attentifs, les historiques jardins d'agrément en question ont souvent été relégués, sur l'échelle des priorités et urgences, bien après la reconstruction de l'habitat et des nécessaires cultures domestiques plus nourricières. Certains nouveaux propriétaires, privés ou publics, ont cependant réaménagé leur pare suivant les modèles propres aux siècles demiers, quelquefois dans une certaine mixité des genres et selon des impératifs financiers.

Enfin, face à la spéculation immobilière et aux ukases de la rentabilité, il a fallu, au cours des dernières décennies, prendre politiquement des mesures de sauvegarde et de conservation, notamment par le classement des pares et des jardins comme sites ou monuments et par la réhabilitation, grâce à de nécessaires mais insuffisantes subventions publiques, la reconstruction (at l'ancienne ou non) occasionnant de nombreux débats. L'ouverture au public, dans une perspective touristique, pose pas mal de problèmes financiers supplémentaires, notamment pour investir dans les structures et le personnel d'accueil et de surveillance, surtout pour les proptiétaires privés. Divers éléments influencent les chances d'équilibrer coûts et recettes : la localisation ou non dans une région qui jouit déjà d'une vocation touristique ; l'accessibilité plus ou moins facile par les moyens de transport privés et collectifs : le cumul d'activités à offrir qui répondent 
à divers centres d'interet (historiques et culturels, sociaux et ludiques, esthétiques ou écologiques), ce qui entraîne la confection d'outils et de processus pédagogiques, la possibilité d'une restauration rapide et démocratique, la nécessité de prévoir des moments d'animation forts et originaux, adaptés à des publics différents-enfants, familles, groupes associatifs, professionnels - et à leur disponibilité suivant le calendrier social et scolaire, les conditions atmosphériques, l'attractivité différente et originale de la flore selon les saisons...

\section{UNE LENTE MATURATION}

La prise de conscience progressive, depuis trois décennies, de la richesse des patrimoines naturels et culturels - héritages de l'histoire du pays ainsi que de son évolution politique et économique, y compris des effets bénéfiques, mais aussi de plus en plus pervers - explique l'émergence à la fois de la demande et de l'offre touristiques dans ce domaine, toutes deux préoccupées de la défense, du sauvetage et de la mise en valeur de ce trésor commun.

C'est pourquoi les opérations promotionnelles du Commissariat général au Tourisme seront polarisées, chaque année depuis 1970, autour d'un thème différent en vue de favoriser / acculturation touristique, comme l'affirme son responsable, Arthur Haulot.

En effet, après l'anné des Châteaux, et celle des abbayes et des béguinages, en 1974, l'année 1976 fut prophétiquement consacrée aux « Paysages, parcs et jardins $x$ : l'allocation inaugurale de la campagne ne justifiait-elle pas cette action indispensable en raison de l'ignorance dont ce patrimoine était l'objet à cette époque?

Cette initiative inedite eut un enorme retentissement dans la presse nationale et internationale, notamment, au Québec. dans Le Jow, La Presse, le Journal de Montreal ou Le Devoir d'Ottawa! Elle a provoqué une mobilisation enthousiaste et décentralisée de la part des écoles primaires et des acteurs culturels : musées, cinémathèque nationale, radio et télévision mirent sur pied expositions, semaine du film et émissions autour du sujet privilégié. Les organismes touristiques publics, (10caux ou provinciaux) et associatifs $y$ contribuèrent largement : publications, concours, rallyes et promenades.
A noter, parmi les manifestations exceptionnelles et significatives : exposition de 100000 roses dans la roseraie du parc appartenant au domaine des princes de Croy-Roeulx ; tapis de fleur's sur la grandplace de Bruxelles ; réouverture du Palais des plantes au Jardin botanique national de Meise ; centième anniversaire du Cercle horticole d'Izegem ; Journé nationale d'hommage rendu aux 600 forestiers rassemblés afin de sensibiliser le public à leur rôle de gardiens de ce patrimoine qui s'ouvre aux touristes et aux services éducatifs qu'ils peuvent rendre.

Une attention plus particulière sera accordée aux « Jardins Historiques $*$; exposition de photos et de documents sur les jardins historiques à la Gare Centrale de Bruxelles ; exposition de photos et de documents sur le Parc de Bruxelles, son histoire et son animation, dans une salle à proximité du parc ; livre de René Pechère, président de l'Association nationale des architectes-paysagers ainsi que de l'Association des Parcs et Jardins de Belgique, révélant la vingtaine de jardins historiques sélectionnés pour cette année thématique ; table ronde, à l'initiative du Commissa= riat général au Tourisme, intitulée « Présent et avenir des châteaux, parcs et jardins $\%$, réunissant des représentants des pouvoirs publics, de nombreux propriétaires privés de domaines concernés, ainsi que des spécialistes Belges et étrangers.

De plus, le Comité international des jardins et sites historiques a profité de l'événement pour organiser à Bruxelles un colloque scientifique ayant pour but de définir les conditions de survie de ce patrimoine menacé. Les organisations internationales ICOMOS et IFLA s'étaient associées pour fonder celui-ci, en 1971, afin que puissent travailler en commun historiens de l'art, architectes et paysagis= tes, archéologues et botanistes, pour étudier et sauvegarder ces témoins particuliêrement fragiles. En conséquence, lors de leur rencontre biennale de 1981 a Florence, ils décidèrent d'élaborer une Charte relative à la conservation et à la valorisation des jardins historiques, qui portera le nom de cette ville et complétera la Charte de Venise d'Icomos consacrée au patrimoine immobilier. Ceux-ci sont définis comme une composition architecturale et végétale qui présente, au point de vue de l'histoire de l'art, un intérêt public.
Commentant cette définition, Henri Chesnot rappelle donc les Nouvelles du Patrimoine ( ${ }^{\circ} 15$, p. 3-4) que

\begin{abstract}
par sa matière celui-ciest un patrimoine vivant, fruit de la composition humaine avec la nature pour base. Il est par conséquent en constante mutation et ce rapport homme/nature forme whe synthèse où s'expriment à la fois tous les arts : l'architeclure par le rappont de l'espace et des masses vegetales, la scalpture par l'art topiaire, la peinture avec la mise en place de tableaux et de couleurs, la musique par le rythme des plantations ef le son de l'eau qui coule et jaillit, et la poésie par les combinaisons sans cesse différentes de ces éléments au cours de la promenade... De plus, les jardins historiques nous intéressent en tant qu'expression des rapports de Thomme, de la société avec la nature et de leur évolation. Depuls les origines, le jardin est wn microcosme évoquant au sens etymologique la notion de "paradis", un monde idealise ou l'homme retrouve grace a son ingéniosité ce qu'il imagine être le lieu originel. Cette évolution permet un autre regard sur notre civilisation dont le jardin est le rejlet.
\end{abstract}

Un pas de plus est franchi lorsque la Commission des Communautés Européennes (C.E.E.), dans le cadre de sa direction Action Culturelle, décida de structurer ses activités autour de thèmes annuels. $\mathrm{Si}_{4}$ en 1992 , les responsables voulurent valoriser des projets de conservation visant à réhabiliter, selon une approche intégrée, le monument et ses abords dans l'espace public environnant, en 1993, le theme du concours était les jardins historiques. Ce terme \& $s$ 'applique aussi bien aux jardins modestes qu'aux parcs ordonnancés aux paysages, aux jardins botaniques, aux cloîtres, aux vergers et aux espaces verts situés dans les centres historiques \%.

En echo à l'initiative européenne, la région wallonne a choisi comme fil conducteur des cinquièmes Journées du Patrimoine organisées au cours du week-end des $11-12$ septembre 1993, dans le cadre du Conseil de l'Europe, les parcs et jardins de Wallonie entourant les grands monuments publics ou privés qui vont s'ouvrir à la visite et a la découverte : 
Le programute comporte plus de 600 activités principales et plus de 300 animations ponctuelles reparties dans les cinq provinces wallonnes. A cote des ouvertures classiques de bâtiments publics ou privés, de chantiers de restauration ou de foulles, nombre de parcs, jardins, cloîtres, serres réserves naturelles... sont pour la première fois inscrits au programme.

A noter qu'un film de treize minutes « Histoire de jardins \%, réalisé conjointement par la Lorraine française, le Grand Duché du Luxembourg et la région wallonne, dans le cadre des collaborations transfrontalières, sera présenté dans les Jardins d'Annevoie. Complémentairement, deux ans plus tard, soit en 1995, la Région de Bruxelles-Capitale catalysera les activités des Journếes du Patrimoine autour du thème \& Architecture et Nature w : a La richesse et la diversité de l'impressionnant patrimoine vert de Bruxelles seront mis en évidence. Du site semi-naturel au jardin architecturé, du parc public à la verdure des intérieurs d'îlots, rien ne serạ oublié... la nature policée trouvera en l'architecture un cadre qui la met en valeur ou qui est son obligé $\%$.

\section{UN ÉCHANTILLON RÉVÉLATEUR}

Les quelques exemples suivants illustrent la diversité et la richesse des offres touristiques. La récente réédition du Guide des Jardins en propose près de 350 , classés géographiquement, par province et localité ! De son côté, le Guide des Attractions et Musées de Belgique 1999, source des extraits de ce chapitre, n'en mentionne qu'une quinzaine (membres cotisants de leur association d'intérêt et répondant à leurs critères propres) dans la rubrique intitulê a Jardins, parcs et rếserves naturelles $\%$. La présentation du livre sur « Tous les Jardins du monde $s$, reprise au dos de la jaquette arrière, suggère une classification de type historique. Sur près de trois millếnaires,

tous les jardins soumettent la nature à leurs artifices : compositions architecturales à Rome ou jardins clos du Moyen Age, theatres de l'humanisme à la Renaissance et reflets des monarques du Grand Siecle, peinures de paysages en Angleterre, pro- menades publiques dans l' Europe entiere, urbanisme contemporain enfin. L'art des jardins est lie au cultures et aux sociétés quil'imaginent.

Une autre approche, davantage préoccupếe d'animation socioculturelle et touristique, sera attentive aux centres d'intérêt et aux aspirations des futurs visiteurs.

\section{JARDINS DE PLAISANCE}

Dans la première catégorie de jardins historiques du pays se trouvent ceux qui agrémentent la vie de châteaux : 85 sont repris dans le Guide des Jardins. Au choix, en Province de Namur et en bordure de Meuse : l'un, « Au cour d'un écrin de 12 ha, dans une atmosphère de beauté et de raffinement, appartenant à la Société Servais-Montpellier, les jardins du château d'Annevoie, chef-d'cuvre de l'art des jardins du XVIII ${ }^{c}$ siècle et dont les jeux d'eau, de renommée internationale, font l'originalité du domaine, classé patrimoine majeur de Wallonie \& ; I'autre, \& Propriété de la famille Bonaert, jardins classiques les plus français de Belgique, avec leurs orangers tricentenaires et leurs orangeries les plus vieilles du pays, dans le domaine de Freyr, à Hastière, agrémentant la demeure Renaissance, agrandie en résidence ducale au XVIII ${ }^{c}$ siecle $\%$.

Autre cas de figure, le Domaine provincial Valéry Cousin, à Chevetogne, tente de répondre à la demande de produits touristiques verts de qualité, liée au développement des préoccupations environnementạles. Il regroupe, autour du château, des etangs paysagers et une futaie jardinée. Pour permettre aux visiteurs, enfants el adultes, de s"initier à l'art de a construire avec la nature $\%$, six jardins ont été créés, à parcourir à pied, un petit train gratuit assurant le retour : le jardin à la française avec une roseraic qui tente d'imposer à la nature un ordre humain ; le jardin de sousbois, woodland-garden anglo-japonais, qui essaie plutôt d'imiter la nature ; la charmille qui enferme et retient les senteurs : les jardins des Licornes; la Folie des Ronces et, enfin, le jardin des plantes médicinales qu'accompagne un livre illustré pour enfants, ayant celui-ci pour cadte. Les hébergements de divers types favorisent notamment des séjours pédagogiques axés autour des problématiques concernées par un environnement durable. Enfin, l'émission d'éducation et de sensibilisation grand public a Jardins et Loisirs *, de Luc Noẻl, à la Télévision francophone RTBF, y a planté ses caméras dans le but de prodiguer des conseils et de faire la démonstration, sur le terrain, de l'état successif des diverses floraisons.

Parallèlement, en Province du Hainaut,

le château de Belail est la propriété et la residence privele des Princes de Ligne depuis six siècles, avec ses collections d'objets d'art et sa célebre bibliotheque : wn pent train fait traverser le parc de 25 ha da XVIIK siecle, constituant un des plas beaw jardins à la française d'Europe.

Rivalisant avec celui-ci et appartenant à la Communauté française.

le Musee Royal de Mariemont, à Morlanwelz, aux célebres collections d'art antique et de porcelaines de Toumai, offre un parc splendide où, parmi les arbres, pelouses et flewrs, un ensemble unique de bronzes monumentau japonais et de sculp. tures belges des $X I X^{c}-X X^{c}$ siecles jouxlent les ruines romantiques du château de Charles de Lorraine et comprenant aussi fontaines, roseraie, orangerie, parc d'hiver et potager...

\section{JARDINS MYSTIQUES}

De leur côté, les grandes abbayes rurales, outre leurs potagers et leurs espaces fruitiers, ont développé des jardins à vocation religieuse en faveur de la méditation personnelle, au sein de la clôture et habituellement enfermés par un cloitre couvert. Parmi les plus anciens qui ont été conservés,

les jardins de style classique de I'ancienne Archicommanderie de I'ordre Teuronique. moines à vocation militaire, fondé à Alden Biesen en 1220, en Province du Limbourg, et actuellemeni devenue un Centre culturel de la Communaté flamande, peavent être visitếs, moyennant paiement, durant la saison touris. tique. Par contre, la promenade le long des batiments, la visife de l'église et du parc de style anglais sont gratwites. 
De même, sur le site de l'ancienne abbaye des Chanoines réguliers de Prémontrés, fondée à Heylissem en 1129 , le domaine provincial d'Hélecine, en Brabant wallon, a pris une option nettement touristique, le château du XVIII étant utilisé comme centre de seminaires, de sejour et de réception ; en outre, le parc de 28 ha comprend une réserve naturelle, un cadran solaire géant, une glacière du XIXe siecle, des étangs de pêche ainsi que des aires de sport, de jeu et de restauration... s.

Enfin, l'ancienne abbaye cistercientie de Cambon-Casteau, fondée le long de lat Dendre au XII siècle, en province du Hainaut, reconstruite au XVIII ${ }^{*}$ avait été detruite par la Révolution française et abandonnée en ruines durant 200 ans. Le parc ornithologique Paradisio y a aménagé la plus grande volière d'Europe avec 2500 oiseaux en semi-liberté, notamment des volatiles exotiques dans la serre tropicale et le jardin d'orchidées. Le par'c de l'abbaye comprend en outre des arbres centenaires, des étangs, un verger, un jardin aromatique, une ferme pour enfants avec des animaux domestiques indigènes ainsi que des restaurants, et une plaine de jeux vaste et originale : une crypte raconte et illustre l'histoire de ce monastere dont témoigne d'ailleurs le cimetiêre des moinés, à visiter.

Le Guide des Jardins invite à découvrir aussi : le jardin de terroir et son pare a la française de 10 ha, réaménagés avec prouesse par une famille privée et des architectes paysagers du Bureau Pochere. sur le site de lancienne abbaye de GrandPré, aninsi que les Jardins de l'abbaye Notre-Dame du Vivier, aménagés selon les conseils des mêmes specialistes, ht Marche-les-Dames, tous deux en province de Namur ; les Jardins de l'ancienne abbaye cistercienne d'Aywiers, cré6e au XII ${ }^{2}$ siecle, en Brabant wallon, dont le pare de style anglais âté restauré au $X I X^{2}$ et les jardins, depuis 1987, où les propriétaires privés organisent en mai et septembre un week-end des plantes et du jardin. Le Guide recommande, en outre, le Jardin de curé, recréé par le célèbre paysagiste Jacques Wirtz, à Kapellen, en province d'Anvers, ainsi que les Jardins de l'Évểché de Namur, ancien refuge de l"abbaye de Malonne, et le Jardin rénové du Palais episcopal de Tournai, au pied de la cathédrale. Enfin, on y lit que le regret que les développements récents dans le parc emmuré de la Maison du Chapitre de chanoines, jouxtant la collégiale de Hoegaarden en Branbant flamand, pour y accueillir une exposition permanente d'une vingtaine de jardins thématiques organisée par des paysagistes et des entrepreneurs de jardins, n'aient pas respecté le caractère typique de jardin clos de communautés religieuses alors qu'on y rend service aux clients interessés.

\section{JARDINS BOTANIQUES}

Dans une dénarche encyclopedique d'étude et de classification des plantes, des jardins botaniques ont rempli cette mission scientifique : celui de Montpellier, cree par Henri IV, en France, ou de Leyde, en Hollande. Ils ont fêté tous deux, en 1993, leur $400^{\circ}$ anniversaire. A Bruxelles. la Société Royale d'Horticulture des PaysBas, soutenue par le roi Guillaume $1^{e r}$, a inauguré le Jardin Botanique actuel, en 1829, durant l'occupation hollandaise. La rotonde centrale et les ailes latérales, de l'architecte Sluys, sous l'aspect traditionnel d'orangerie, sont largement vitrées comme une serre. Racheté par l'Etat en 1870, le Jardin est devenu, en 1984, un Centre culturel dynamique et irremplaçable de la Communauté française, agrémenté $\mathrm{d}^{+}$un parc public récemment restauré, elément constitutif et imprenable du paysage bruxellois dans le haut de la ville. La plupart des collections qui, fin du siecle. comprenaient plus de 7000 plantes, ont été transférées dans le Jardin Botanique National de Belgique, au Domaine de Bouchout, à Meise, acquis par l'État en 1938, avec jardin de plein air et serres tropicales du Palais des plantes. A Liège, les collections ont égallement quitté le Jardin botanique du Centre-ville (1887) pour se joindre à l'Institut de Botanique sur le nouveau site de l'Université du SrtTilman où celui-ci anime l'Observatoite du Monde des Plantes, de divers continents, dans de vastes serres, Enfin, les progrès techniques de la fin du siecte dernier, dans l'architecture en fer et en verre, ont permis d'edifier les remarquables Serres Royales de Laeken, voulues par le roi Léopold II et réalisées par I'architecte Ballat (1891); elles abritent des especes tropicales et sont entretenues par une vingtaine de jardiniers.

Parmi la dizaine d'arborétums du royaume, les plus célèbres sont celui du Domaine provincial de Bokrijk, qui abrique le musée de plein air sur l'architecture populaire. et celui du Musce Royal de 1'Afrique centrale a Tervuren.

\section{PLANTES MÉDICINALES}

Au Moyen Age, des * femmes savantes * des curés et la plupart des monastères cultivaient les plantes médicinales dans un a jardin des simples $w$. L'abbaye trappiste d'Orval, en province du Luxembourg, possède encore le sien couplè à un musée pharmaceutique, De son côté, l'Association pour la Promotion Touristique et Culturelle de I'ancienne abbaye cistercienne de Villers-lä-Ville, en Brabant wallon (APTCV), travaille en permanence à la restauration et au réaménagement pédagogique du jardin des plantes médicinales que l'on peut visiter sur le site des ruines en même temps que le Jardin du Palais de l'Abbe. A noter que les jardins botaniques mentionnes plus haut comportent chacun une section consacré à ce domaine spécifique. C est leur dimension thérapeutique quil les rattache aux facultês de médecine des diverses universités du palys.

\section{JARDINS OUVERTS}

Pres de 150 proprićtaires de jardins privés ont accepté de figurer dans le Guide des Jardins afin de partager leur passion, de favoriser les échanges entre eux et de prodiguer des conseils aux nouveaux ou aux futurs pratiquants. L'affluence de plus en plus importante aux Journées du Patrimoine auxquelles ils contribuent, les demandes d'interviews de plus en plus nombreuses de la part des médias, la multiplication de a Rencontres Jardinieres $n$ entre professionnels, entreprises spécialisées et amateurs éclairés... les ont convaincus. La plupart font partie du réseau Jardins Ouverts : tout citoyen qui devient membre de l'association peut visiter ces derniers, ă l'aide du catalogue qui lui fournit toute l'information utile.

\section{PARCS PUBLICS}

Le concept de parc public, ouvert à la population, a de lointaines origines, notamment dejà dans les $\alpha$ capitulaires carolingiens $*$ qui ordonnaient la plantation de jardins publics pour l'agrément. En effet, autour de l'an mil, de telles realisations existaient au Prado en Espagne, au 
Prater à Vienne, à Saint-Germain-des-Prés et au Pré-aux-Cleres à Paris. Mais c'est le $X I X$ siecle surtout qui préconise la création de jardins publics, envisagés comme outils de réforme sociale, d"abord en Angleterre puis à Paris dans le cadre du plan d'urbanisme de Napoléon III pour restructurer sa capitale, avec la collaboration du baron Haussman. C'est dans cette optique qu'il donne, en 1852, le Bois de Boulogne, ancienne forêt royale, pour en faire un lieu de délassement public.

L'ensemble des pays d'Europe suivent ce mouvement. Citons, particulièrement en Belgique, le roi $*$ urbaniste $*$ et bâtisseur Léopold II, qui contribue à ce que Bruxelles devienne une des capitales les plus vertes du monde, avec, entre autres, le Parc de Bruxelles, situé entre le Palais royal et le Parlement fédéral, le pare du Cinquantenaire où se trouve le Musée Royal d'art et d'histoire, le Bois de la Cambre comme parc paysager, le parc de la Woluwe à l'initiative du roi dans le cadre de l'Exposition universelle de $1897 \mathrm{et}$, enfin, les espaces verts classiques des anciennes abbayes, les cisterciennes de la Cambre, les augustins du Rouge Cloître et les prémontrés de Dieleghem.

A noter également, à l'ombre de l'ancien Chapitre de la collégiale Saint-Guidon, à Anderlecht, le jardin de la Maison d'Érasme, ecclésiastique humaniste accueilli dans la maison canoniale d'un ami chanoine. Enfin, le Centre régional d'initiation à l'écologie * Toumesol * est abrité dans le parc de l'ancienne grande famille bourgeoise et industrielle Tournay-Solvay racheté par la région de Bruxelles capitale en 1980 . Toutes les grandes villes du pays bénéficient de joyaux semblables: a Anvers, le jardin de la Maison Rubens ou le parc Middelheim, devenu musée permanent de sculpture en plein air et thêture de la biennale internationale d'art contemporain, sans oublier le Jardin zoologique qui est d'abord un magnifique pare paysager aux parterres saisonniers de fleurs et aux grands arbres d'essences autochtones et exotiques. Malines peut s'enorgueillir du parc Tivoli que la ville a racheté en 1979 , lui confiant une vocation éducative et ludique : roseraie, plantes médicinales, arbres fruitiers (considérés comme conservatoire des anciennes espèces et géré par la Fondation nationale des vergers), ferme pour les enfants. En région wallonne, il est assez inattendu qu* un petit village comme Villers-Sainte-Gertrude possède un parc public, situé au sein du domaine voué au tourisme rural de séjour et géré par la Communauté française, comportant un arboretum qui met en valeur les arbres centenaires. De même, le village d'Enghien a racheté, en 1985 , le château et le parc appartenant aux ducs d Arenberg : le parc a été aménagé au XVII siêcle par l'un d'entre eux, aidé de oncle, le père capucin Antoine, et il constitue un cadre unique pour un ensemble d'animations socioculturelles et récréatives.

\section{TOURISME ÉCOCULTUREL ?}

Tout ce qui précède ne rend-il pas pertinent ce qu'affirme l'ouvrage sur le Tourisme Culturel ?

\section{Il s'agit là d'un tourisme motivé par les espaces naturels appréhendés non seulement du point de vue de leurs paysages, de leur flore ou de leur faune, mais également de leur histoire : c'est la culture de la na- ture. L'objet de la demarche induite par le concept est de nenfoncer a les- prit du lieu et de mettre en exer- gue l'ensemble de ses significations, qu'elles soient de l'ordre de la na- ture ou de la culture, de la géogra- phie ou de l'histoire... En France les espaces de pure nature inviolé sont rares, les paysages sont whe nature jardinee.}

Bientôt, l'annếe 2000 va permettre d'exercer ce genre de tourisme au maximum. En effet, elle célćbrera d'abord le tricentenaire de la mort du pionnier européen de l'art des jardins, André Lenôtre (1613-1700). qui passa la majeure partie de sa vie à apprendre le métier de jardinier, à étudier les jardins et ả concevoir l'adéquation entre nature et architecture, les Jardins de Versailles étant le chef-d'auvre de cet apprenti de génie !

Il est significatif et symbolique que, vingtcing ans plus tard, l'Office de Promotion du Tourisme (OPT) de la Communauté française ait choisi le meme thème annuel que le Commissariat general au Tourisme 1976 pour démarrer le troisième millénaire: la boucle est bouclée, le Paradis nouveau est annonce.

André Hut est journaliste, spécialisé en ani. mation touristique.

\section{BIBLIOGRAPHIE}

Association Attraction at Tourisme (1999), Guide des Atractions touristiques et Musér de Belgique, a/s Grottes de Han, B. 5580 Han-surLesse, distribution gratuite dans les Bureaux d'information touristique.

Association des amis de l'UNESCO, Nouvelles du Parrimoine, Bimestriel, avenue Général de Gaulle, 17, 1050 Bruxelles, tél. : 02/648.80.06.

Les Jardins Historiques, n" 15, juillet/août 1987 : Les Serres, n² 20, mai 1988 : Paysages et Patrimoine, $\mathrm{n}^{\circ}$ 39. juin 1991 ; Historique ; Degembe Marie-Françoise, $\mathbf{n}^{\circ} 47$, decembre 1992, $\mathrm{n}^{\circ} 51$. septembre 1993, $\mathrm{n}^{\circ} 58$, novembre $1994 \ldots$

de Groote, Christine (1997), Le Guide des Jardins de Belgique, Editions Racine, Bruxelles.

Domaine Provincial de Chevelogme (1998), Plan du domaine. Présentation du Sentier des Jardins, B. 5590 Chevetogne.

Donnay, Guy (1993), Guide du Parc et du Musée de Mariemont, Musée Royal de Mariemont, Chaussée de Mariemont, 100, B. 7140 Morlanwelz.

Duquenne, Xavier (1993), Le Botanique de 1829 a nos jours, Collectif, 1993 ; Le Pare de Bruxelles, CFC-Éditions, Boulevard de Waterloo, 102 , 1000 Bruxelles.

Institut Bruxellois pour la Gestion de l'Environnement (1996), Decouvrir Bruxelles par ses Espaces Verts, IBGE, Région de BruxellesCapitale, Gulledelle, 100, 1200 Bruxelles.

Institut Bruxellois pour la Gestion de l'Environnement, Dépliants Narure et Parcs, Série commencée depuis 1997, tél. : 02/775,75,75.

Jardins Ouverts, ASBL, Catalogue 1998, Chaussée de Vleurgat, 108, 1000 Bruxelles, tél/fax: $02 / 646.97 .36$

Office de Promotion du Tourisme, OPT, rue du Marché aux Herbes, 61, 1000 Bruxelles, tél.. : 02/504,02.00, fax : 02/513.69.50.

Origet du Cluzeau, Claude (1998), Le Tourisme Culhurel, Editions PUF, Collection Que Sais-Je ? 3389 , Paris.

Pechère, René (1976), Pancs et Jardins de Belgique, Éditions Rossel, Bruxelles.

Pechère, Rene (1995), "Grammaire des jardins, Secrets de métier *. Pares ef Jardins de Belgique. Éditions Rossel, Bruxelles.

Van Zuylen, Gabrielie (1994), Tous Les Jardins du Monde, Éditions Découverte Gallimard, collection Art de Vivre, 207, Paris. 